\title{
Minerais do grupo da crichtonita em veios de quartzo da Serra do Espinhaço (Minas Gerais e Bahia)
}

\author{
Minerals of the crichtonite group in quartz veins from the Espinhaço Mountain Range \\ (States of Minas Gerais and Bahia, Brazil) \\ Mario Luiz de Sá Carneiro Chaves ${ }^{1}$ e Luiz Alberto Dias Menezes Filho² \\ ${ }^{1}$ Universidade Federal de Minas Gerais - UFMG, Instituto de Geociências, Centro de Pesquisa Prof. Manoel Teixeira da Costa, \\ Avenida Antonio Carlos, 6627, CEP 31270-901, Belo Horizonte, MG, Brasil (mlschaves@gmail.com) \\ 2Luiz Menezes Minerals, Belo Horizonte, MG, Brasil (In Memoriam)
}

Recebido em 7 de março de 2016; aceito em 10 de agosto de 2016

\begin{abstract}
Resumo
O trabalho descreve quatro minerais do grupo da crichtonita encontrados em veios hidrotermais de quartzo, que cortam diversas formações geológicas da Serra do Espinhaço (Supergrupo Espinhaço). Esses minerais - senaíta, crichtonita, almeidaíta e gramacciolíta-(Y) - foram coletados em duas regiões: Presidente Kubitschek, ao sul de Diamantina (MG), e Novo Horizonte, na Chapada Diamantina Ocidental (BA). A idade de tais veios é relacionada ao final do Ciclo Brasiliano, em ca. 490 Ma. A senaíta é um mineral descoberto no Brasil e classicamente identificado nos aluviões diamantíferos da região de Diamantina; sua primeira ocorrência primária é aqui descrita. A crichtonita, apesar de conhecida desde longa data, teve seu primeiro depósito no país reportado nessa mesma região. A almeidaíta é o $61^{\circ} \mathrm{e}$ um dos mais recentes dos "minerais brasileiros", sendo encontrada na região de Novo Horizonte. A gramaccioliíta-(Y), uma espécie descoberta na última década (Itália), tem sua primeira ocorrência brasileira descrita também nessa região. Os depósitos e as composições químicas desses minerais são caracterizados, bem como as problemáticas envolvidas até suas definições finais.
\end{abstract}

Palavras-chave: Grupo da crichtonita; Veios de quartzo; Serra do Espinhaço.

\begin{abstract}
Four minerals of the crichtonite group found in hydrothermal quartz veins that cross several units of the Espinhaço Supergroup in the Espinhaço Mountain Range are described in this paper. These minerals, senaite, crichtonite, almeidaite and gramaccioliite-(Y), were collected in two regions: Presidente Kubitschek, south from Diamantina (State of Minas Gerais), and Novo Horizonte, in the Western Diamantina Tableland (State of Bahia). The age of these veins are related with the end of Brasiliano Cycle, at ca. $490 \mathrm{Ma}$. Senaite is a mineral discovered in Brazil and is usually recognized in diamond-bearing alluvial deposits of the Diamantina region. Its occurrence as a primary mineral in situ is for the first time described here. Although crichtonite has been known for quite a long time, its first occurrence in Brazil has been reported in the same region in Minas Gerais. Almeidaite is the $61^{\text {th }}$ "newest Brazilian mineral" and has been found in the region of Novo Horizonte. Gramaccioliite-(Y), a species discovered in the last decade in Italy, has also been described for the first time in Novo Horizonte, Bahia. The deposits and the chemical compositions of these rare minerals are characterized in this paper, as well as the problems involved until their final recognition.
\end{abstract}

Keywords: Crichtonite group; Quartz veins; Espinhaço Mountain Range. 


\section{INTRODUÇÃO}

O grupo da crichtonita, da classe dos óxidos, abrange atualmente 17 minerais: almeidaíta, cleusonita, crichtonita, davidita-(Ce), davidita-(La), davidita-(Y), dessauíta-(Y), gramaccioliíta-(Y), landauíta, lindsleyíta, loveringita, mapiquiroíta, mathiasita, mianningita, paseroíta, senaíta e "unnamed (HBU UK-4)" (Wülser et al., 2004; Back e Mandarino, 2008; MINDAT, 2016 - Tabela 1). A semelhança física (e menor, química) desses minerais com a ilmenita fez com que estudos a seu respeito ficassem por longo tempo limitados a descrições macroscópicas básicas. Ainda assim, a senaíta foi o segundo mineral identificado do grupo, a partir de amostras de concentrados de minerais pesados de aluviões diamantíferos da região de Diamantina, Minas Gerais (Hussak e Prior, 1898). Esse fato fez com que o mineral fosse considerado um dos satélites "clássicos" do diamante em tal região (Hussak, 1917; Guimarães, 1934).

A Serra do Espinhaço constitui uma cadeia montanhosa que se estende de sul para norte, desde a porção leste do Quadrilátero Ferrífero, região central de Minas Gerais, até o limite entre os estados da Bahia e Piauí, por cerca de $1.200 \mathrm{~km}$ naquela direção (Figura 1). A unidade litoestratigráfica que edifica a serra é o Supergrupo Espinhaço, constituído por metassedimentos e, subordinadamente, rochas metavulcânicas cuja deposição ocorreu no Paleo-Mesoproterozoico (Machado et al., 1989; Dussin, 1994; Chemale Júnior et al., 2012). O objetivo deste trabalho é descrever a mineralogia e a geologia dos depósitos dos quatro minerais do grupo da crichtonita encontrados nessa serra. Tais minerais - senaíta, crichtonita, almeidaíta e gramacciolíta-(Y) - associam-se primariamente a veios hidrotermais de quartzo que atravessam

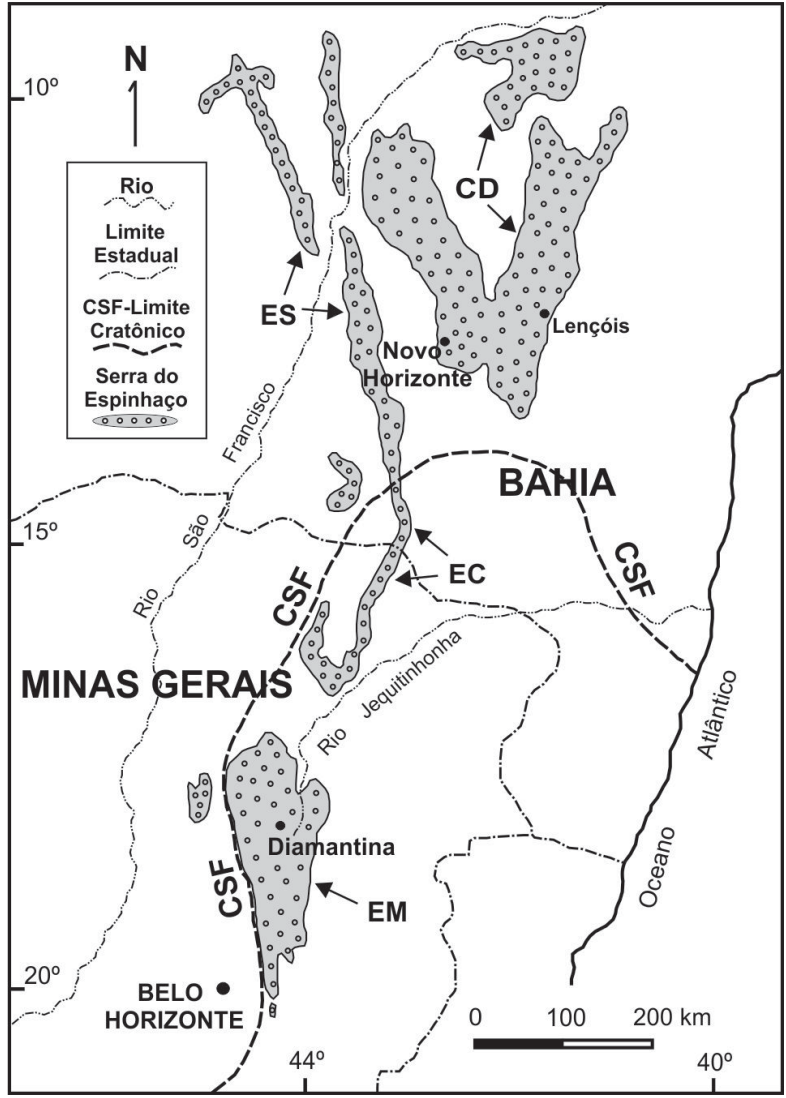

Figura 1. A Serra do Espinhaço em Minas Gerais e Bahia com seus domínios geográficos e geotectônicos, destacando as duas localidades onde foram estudados os minerais do grupo da crichtonita (Diamantina e Novo Horizonte). Domínios: EM - Espinhaço Meridional; EC - Espinhaço Central; CD - Chapada Diamantina; ES - Espinhaço Setentrional.

Tabela 1. Relação e composição química dos minerais do grupo da crichtonita, em ordem alfabética por subgrupo genético (Wülser et al., 2004, modificado). Composições químicas homogeneizadas conforme MINDAT (2016).

\begin{tabular}{|c|c|c|c|}
\hline \multicolumn{2}{|c|}{ Subgrupo Genético } & Mineral & Composição Química \\
\hline \multicolumn{2}{|c|}{$\begin{array}{c}\text { METAMORFISMO } \\
\text { (VEIOS HIDROTERMAIS) }\end{array}$} & $\begin{array}{l}\text { Almeidaíta } \\
\text { Cleusonita } \\
\text { Crichtonita } \\
\text { Dessauíta-(Y) } \\
\text { Gramacciolíta-(Y) } \\
\text { Senaíta }\end{array}$ & $\begin{array}{l}\mathrm{PbZn}_{2}(\mathrm{Mn}, \mathrm{Y})\left(\mathrm{Ti}, \mathrm{Fe}^{3+}\right)_{18} \mathrm{O}_{37}(\mathrm{OH}, \mathrm{O}) \\
(\mathrm{Pb}, \mathrm{Sr})\left(\mathrm{U}^{4+}, \mathrm{U}^{6+}\right)\left(\mathrm{Fe}^{2+}, \mathrm{Zn}\right)_{2}\left(\mathrm{Ti}, \mathrm{Fe}^{2+}, \mathrm{Fe}^{3+}\right)_{18}(\mathrm{O}, \mathrm{OH})_{38} \\
\mathrm{Sr}(\mathrm{Mn}, \mathrm{Y}, \mathrm{U}) \mathrm{Fe}_{2}(\mathrm{Ti}, \mathrm{Fe}, \mathrm{Cr}, \mathrm{V})_{18}(\mathrm{O}, \mathrm{OH})_{38} \\
(\mathrm{Sr}, \mathrm{Pb})(\mathrm{Y}, \mathrm{U})\left(\mathrm{Ti}, \mathrm{Fe}^{3+}\right)_{20} \mathrm{O}_{38} \\
(\mathrm{~Pb}, \mathrm{Sr})(\mathrm{Y}, \mathrm{Mn}) \mathrm{Fe} 3+2\left(\mathrm{Ti}, \mathrm{Fe}^{3+}\right)_{18} \mathrm{O}_{38} \\
\mathrm{~Pb}(\mathrm{Mn}, \mathrm{Y}, \mathrm{U})(\mathrm{Fe}, \mathrm{Zn})_{2}\left(\mathrm{Ti}, \mathrm{Fe}, \mathrm{Cr}, \mathrm{V}_{18}(\mathrm{O}, \mathrm{OH})_{38}\right.\end{array}$ \\
\hline \multirow{3}{*}{$\begin{array}{c}\text { MAGMATISMO } \\
\text { (METASSOMATISMO) }\end{array}$} & GRANITO & $\begin{array}{l}\text { Davidita-(Ce) } \\
\text { Davidita-(La) } \\
\text { Davidita-(Y) } \\
\end{array}$ & $\begin{array}{l}(\mathrm{Ce}, \mathrm{La})\left(\mathrm{Y}, \mathrm{U}, \mathrm{Fe}^{2+}\right)\left(\mathrm{Ti}, \mathrm{Fe}^{3+}\right)_{20}(\mathrm{O}, \mathrm{OH})_{38} \\
(\mathrm{La}, \mathrm{Ce})\left(\mathrm{Y}, \mathrm{U}, \mathrm{Fe}^{2+}\right)\left(\mathrm{Ti}, \mathrm{Fe}^{3+}\right)_{20}(\mathrm{O}, \mathrm{OH})_{38} \\
\left.(\mathrm{La}, \mathrm{Ce}, \mathrm{Na}, \mathrm{Ca}, \mathrm{Pb})\left(\mathrm{Y}, \mathrm{Fe}^{2+}, \mathrm{\square}\right) \mathrm{Fe}_{2+}, \mathrm{Mn}^{2+}\right)_{2}\left(\mathrm{Ti}, \mathrm{Fe}^{3+}, \mathrm{Nb}, \mathrm{Zr}\right)_{18} \mathrm{O}_{38}\end{array}$ \\
\hline & SIENITO & $\begin{array}{l}\text { Landauíta } \\
\text { Mianningita } \\
\text { "Unnamed (HBU UK-4)" }\end{array}$ & $\begin{array}{l}\mathrm{NaMnZn}_{2}(\mathrm{Ti}, \mathrm{Fe})_{6} \mathrm{Ti}_{12} \mathrm{O}_{38} \\
(\square, \mathrm{Pb}, \mathrm{Ce}, \mathrm{Na})\left(\mathrm{U}^{4+}, \mathrm{Mn} \bigcup^{6+}\right) \mathrm{Fe}_{2}{ }^{3+}\left(\mathrm{Ti}, \mathrm{Fe}^{3+}\right)_{18} \mathrm{O}_{38} \\
\mathrm{NaFe}^{2+} \mathrm{Zn}_{2}\left(\mathrm{Ti}, \mathrm{Fe}^{3+}, \mathrm{Nb}_{6}\right) \mathrm{Ti}_{12} \mathrm{O}_{38}\end{array}$ \\
\hline & ULTRABÁSICAS & $\begin{array}{l}\text { Lindsleyíta } \\
\text { Loveringita } \\
\text { Mapiquiroíta } \\
\text { Mathiasita } \\
\text { Paseroíta }\end{array}$ & $\begin{array}{l}(\mathrm{Ba}, \mathrm{Sr})(\mathrm{Zr}, \mathrm{Ca})(\mathrm{Fe}, \mathrm{Mg})_{2}(\mathrm{Ti}, \mathrm{Cr}, \mathrm{Fe})_{18} \mathrm{O}_{38} \\
(\mathrm{Ca}, \mathrm{Ce}, \mathrm{La})(\mathrm{Zr}, \mathrm{Fe})(\mathrm{Mg}, \mathrm{Fe})_{2}(\mathrm{Ti}, \mathrm{Fe}, \mathrm{Cr}, \mathrm{Al})_{18} \mathrm{O}_{38} \\
(\mathrm{Sr}, \mathrm{Pb})(\mathrm{U}, \mathrm{Y}) \mathrm{Fe}_{2}\left(\mathrm{Ti}, \mathrm{Fe}^{3+}, \mathrm{Cr}^{3+}\right)_{18} \mathrm{O}_{38} \\
(\mathrm{~K}, \mathrm{Ba}, \mathrm{Sr})(\mathrm{Zr}, \mathrm{Fe})_{2}(\mathrm{Ti}, \mathrm{Cr}, \mathrm{Fe})_{18} \mathrm{O}_{38} \\
\mathrm{~Pb}, \mathrm{Mn}^{2+}\left(\mathrm{Mn}^{2+}, \mathrm{Fe}^{3+}\right)_{2}\left(\mathrm{~V}^{5+}, \mathrm{Ti}, \mathrm{\square}\right)_{18} \mathrm{O}_{38}\end{array}$ \\
\hline
\end{tabular}


diversas unidades do Supergrupo Espinhaço, datados do final do ciclo orogênico Brasiliano em cerca de $490 \mathrm{Ma}$ (Chaves et al., 2010, 2016).

\section{DESCRIÇÃO DOS MINERAIS E SEUS DEPÓSITOS}

De acordo com Orlandi et al. (1997), a fórmula geral do grupo da crichtonita é: $\mathrm{ABC}_{18} \mathrm{~T}_{2} \mathrm{O}_{38}$, onde " $\mathrm{A}$ " = $\mathrm{Sr}, \mathrm{Pb}, \mathrm{Ca}$, $\mathrm{Na}, \mathrm{Ba}, \mathrm{U}$, Elementos Terras Raras; "B" = Mn, Y, ETR, U, $\mathrm{Zr}$; " $\mathrm{C}$ " $=\mathrm{Ti}, \mathrm{Fe}^{3+}, \mathrm{Cr}$; e "T" = $\mathrm{Fe}^{3+}, \mathrm{Mg}$. Os quatro minerais desse grupo identificados na região da Serra do Espinhaço (Figura 1) ocorrem em veios de quartzo e depósitos secundários associados. Tais veios são, em geral, lenticulares, pouco espessos $(<1 \mathrm{~m})$ e de comprimentos comumente inferiores a $5 \mathrm{~m}$. As primeiras ocorrências primárias de senaíta e de gramaccioliíta-(Y) no Brasil são aqui descritas, bem como se apresentam dados adicionais sobre a química mineral da crichtonita. A almeidaíta constitui um dos mais recentes "minerais brasileiros" (Menezes Filho et al., 2015) e, a respeito, destaca-se uma revisão histórica acerca do conhecimento dessa nova espécie em relação às outras três.

As análises químicas com microssonda eletrônica foram efetuadas no Laboratório de Microanálises do Departamento de Física do ICEX/UFMG (Belo Horizonte-MG). Os padrões utilizados nessas análises, para os quatro minerais, foram os seguintes: estroncianita para $\mathrm{SrO}_{2}$; ilmenita para $\mathrm{TiO}_{2}$ e $\mathrm{FeO}$; rodonita para $\mathrm{MnO}$; $\mathrm{ZnO}$ para $\mathrm{ZnO}$; $\mathrm{YAG}_{\text {para }} \mathrm{Y}_{2} \mathrm{O}_{3}$; $\mathrm{PbO}$ para $\mathrm{PbO}$, e Sc-thortvetita para $\mathrm{Sc}_{2} \mathrm{O}_{3}$ (somente na crichtonita).

\section{Senaíta $\left[\mathrm{Pb}(\mathrm{Mn}, \mathrm{Y}, \mathrm{U})(\mathrm{Fe}, \mathrm{Zn})_{2}(\mathrm{Ti}, \mathrm{Fe}, \mathrm{Cr}, \mathrm{V})_{18}(\mathrm{O}, \mathrm{OH})_{38}\right]$}

Esse mineral foi descrito por Hussak e Prior (1898), cujo nome homenageia o geocientista brasileiro Joaquim Candido da Costa Sena (1852-1919), ex-professor da Escola de Minas de Ouro Preto (composição química conforme MINDAT, 2016). Sua presença foi identificada principalmente nos concentrados de minerais pesados de depósitos aluvionares de diamantes de Datas e Extração (antigo Curralinho), nos arredores de Diamantina (MG).

Metelo (1968) analisou quatro amostras "antigas" disponíveis no Museu de Mineralogia do DNPM (Rio de Janeiro) - localidades Datas, Vargem (Datas), Imbirussu (Santa Quitéria) e Rio Cipó, fornecendo detalhamentos sobre a estrutura cristalina do mineral através de análises de fluorescência e difratometria de raios X. A julgar pelo código DNPM idêntico, Foord et al. (1984) reestudaram essas mesmas amostras (embora não tenham feito nenhuma menção ao trabalho anterior). Juntamente com o estudo de Grey et al. (1976), esses dois últimos artigos encerram os dados mais completos sobre a química mineral da senaíta aluvionar da Serra do Espinhaço (Tabela 2).

Cassedanne (1986) e Cassedanne e Chaves (1990) acreditaram ter encontrado pela primeira vez a senaíta in situ, num veio de quartzo da Fazenda Guariba, próxima a Presidente Kubitschek, ao sul de Diamantina. Na ocasião, porém, as análises comprobatórias limitaram-se ao uso de difratometria e fluorescência de raios X. Estudos adicionais com microssonda eletrônica e LA-ICP-MS efetuados pelo primeiro autor deste trabalho e colaboradores, no entanto, levaram à redefinição de tal espécie como crichtonita conforme descrições apresentadas no próximo item (Chaves, 1992; Foord et al., 1994).

Em 2008, Menezes Filho adquiriu um lote de cristais de quartzo rutilado em Diamantina, em que alguns espécimes continham cristais submetálicos pretos, tabulares e parcialmente corroídos, muito semelhantes ao material encontrado na Fazenda Guariba. Análises de EDS mostraram ser um óxido de $\mathrm{Pb}-\mathrm{Ti}-\mathrm{Fe}-\mathrm{Sr}$ com picos mais fracos de $\mathrm{Mn}$, $\mathrm{Y}$ e $\mathrm{Zn}$. Entretanto, como as raias de $\mathrm{Sr}$ e $\mathrm{Pb}$ têm energias diferentes, a amplitude relativa dos picos não seria suficiente para afirmar que se tratava ou não de senaíta ( $\mathrm{Pb}$ dominante) ou de crichtonita (Sr dominante). A confirmação de senaíta deu-se somente através de análises gerais com microssonda eletrônica (Tabela 2), em conjunto e seguindo as mesmas

Tabela 2. Novas análises com microssonda eletrônica em cinco amostras de senaíta in situ de Presidente Kubitschek Córrego do Arruda (médias de 3-4 spots por grão).

\begin{tabular}{|c|c|c|c|c|c|c|c|c|c|c|c|}
\hline Amostra & SrO & $\mathrm{TiO}_{2}$ & $\mathrm{FeO}\left({ }^{*}\right)$ & MnO & Zno & $\mathrm{Y}_{2} \mathrm{O}_{3}$ & $\mathrm{PbO}$ & $\mathrm{MgO}$ & $\mathrm{TR}_{2} \mathrm{O}_{3}$ & $\mathrm{~V}_{2} \mathrm{O}_{5}$ & Total \\
\hline SEN-312-1 & 1,66 & 61,03 & 22,96 & 2,62 & 0,58 & 1,21 & 7,70 & nd & nd & nd & 97,76 \\
\hline SEN-312-2 & 1,65 & 61,11 & 23,32 & 2,88 & 0,66 & 2,17 & 7,26 & nd & nd & nd & 99,05 \\
\hline SEN-312-3 & 1,54 & 60,47 & 23,59 & 2,54 & 0,54 & 1,49 & 7,27 & nd & nd & nd & 97,44 \\
\hline SEN-312-5 & 1,58 & 61,02 & 24,57 & 2,64 & 0,56 & 1,60 & 6,93 & nd & nd & nd & 98,90 \\
\hline SEN-312-6 & 1,60 & 60,37 & 24,44 & 2,07 & 0,62 & 1,83 & 7,35 & nd & nd & nd & 98,28 \\
\hline Média & 1,61 & 60,80 & 23,78 & 2,55 & 0,59 & 1,66 & 7,30 & nd & nd & nd & 98,29 \\
\hline SEN-H (") & nd & 51,21 & 24,48 & 14,06 & nd & nd & 10,24 & nd & nd & nd & 99,99 \\
\hline SEN-G (") & 0,20 & 58,68 & 24,73 & 4,08 & nd & 0,86 & 9,21 & 0,02 & 0,13 & 0,70 & 98,61 \\
\hline SEN-F (") & 1,10 & 54,88 & 25,48 & 3,33 & 0,53 & 3,55 & 9,08 & 0,15 & 1,25 & nd & 99,35 \\
\hline
\end{tabular}

$\left({ }^{\star}\right)$ todo ferro contido como Fe ${ }^{2+} ;\left(^{* *}\right)$ análises anteriores de senaíta aluvionar respectivamente de Hussak e Prior (1898) - média de 4 análises, Grey et al. (1976) e Foord et al. (1984) - médias de 4 análises; nd - não determinado. 
rotinas sobre amostras disponíveis de todos os minerais do grupo.

Também se observa na Tabela 2 que, embora as análises originais de Hussak e Prior (1898) provavelmente continham distorções significantes, já que os métodos analíticos utilizados na época eram mais limitados, foram adequadas o suficiente para caracterizar o novo mineral. Nos novos dados ora obtidos para a senaíta primária, observam-se os valores relativamente mais elevados de $\mathrm{TiO}_{2}$, bem como mais baixos de $\mathrm{FeO}, \mathrm{MnO}$ e $\mathrm{PbO}$ em relação aos apresentados por Grey et al. (1976) e Foord et al. (1984). Com base nesses dados, a fórmula empírica calculada do mineral é $\left(\mathrm{Pb}_{0,59} \mathrm{Sr}_{0,29}\right)_{\Sigma 0,88}\left(\mathrm{Mn}_{0,65} \mathrm{Y}_{0,27}\right)_{\Sigma 0,92}\left(\mathrm{Fe}^{2+}{ }_{1,87} \mathrm{Zn}_{0,13}\right)_{\Sigma 2,00}$ $\left(\mathrm{Ti}_{13,95} \mathrm{Fe}^{3+}{ }_{4,05}\right)_{\sum 18,00} \mathrm{Fe}^{2+}{ }_{0,15} \mathrm{O}_{38}$.

Após essa descoberta, a procedência do material foi levantada, localizando-se a somente $2 \mathrm{~km}$ a nordeste da sede do município de Presidente Kubitschek, nas proximidades do Córrego do Arruda (Figura 2A), sendo visitada pelos autores em 2013 (GPS-UTM 653286/7941934). A geologia da área foi previamente estudada por Uhlein e Garcia (1987), reconhecendo-se filitos pertencentes à Unidade Inferior da Formação Sopa-Brumadinho, nas proximidades do contato com quartzitos e conglomerados da Unidade Média dessa mesma formação (Figura 2B). O mineral ocorre num enxame de veios de quartzo de larguras entre $30-50 \mathrm{~cm}$, subverticais (Figura 2C), contendo "buchos" de forma ovoide com até $1 \mathrm{~m}$ de diâmetro (Figura 2D), onde os únicos minerais acessórios observados foram rutilo e senaíta. $\mathrm{O}$ veio foi explorado nos anos de 2006-2008 e, desde então, os serviços encontram-se inteiramente paralisados.

\section{Crichtonita $\left[\mathrm{Sr}(\mathrm{Mn}, \mathrm{Y}, \mathrm{U}) \mathrm{Fe}_{2}(\mathrm{Ti}, \mathrm{Fe}, \mathrm{Cr}, \mathrm{V})_{18}(\mathrm{O}, \mathrm{OH})_{38}\right]$}

Descrita no início do século 19, em St. Christophe-en-Oisans, Rhône-Alpes, na França (J. Bournon, 1813, de acordo com Back e Mandarino, 2008), essa espécie foi posteriormente encontrada em diversos outros países, como Áustria, Canadá, Estados Unidos, Itália, Madagascar, Rússia e Suíça (MINDAT, 2016). No Brasil, conforme ressaltado no item anterior, sua primeira descrição em Presidente Kubitschek deve-se a
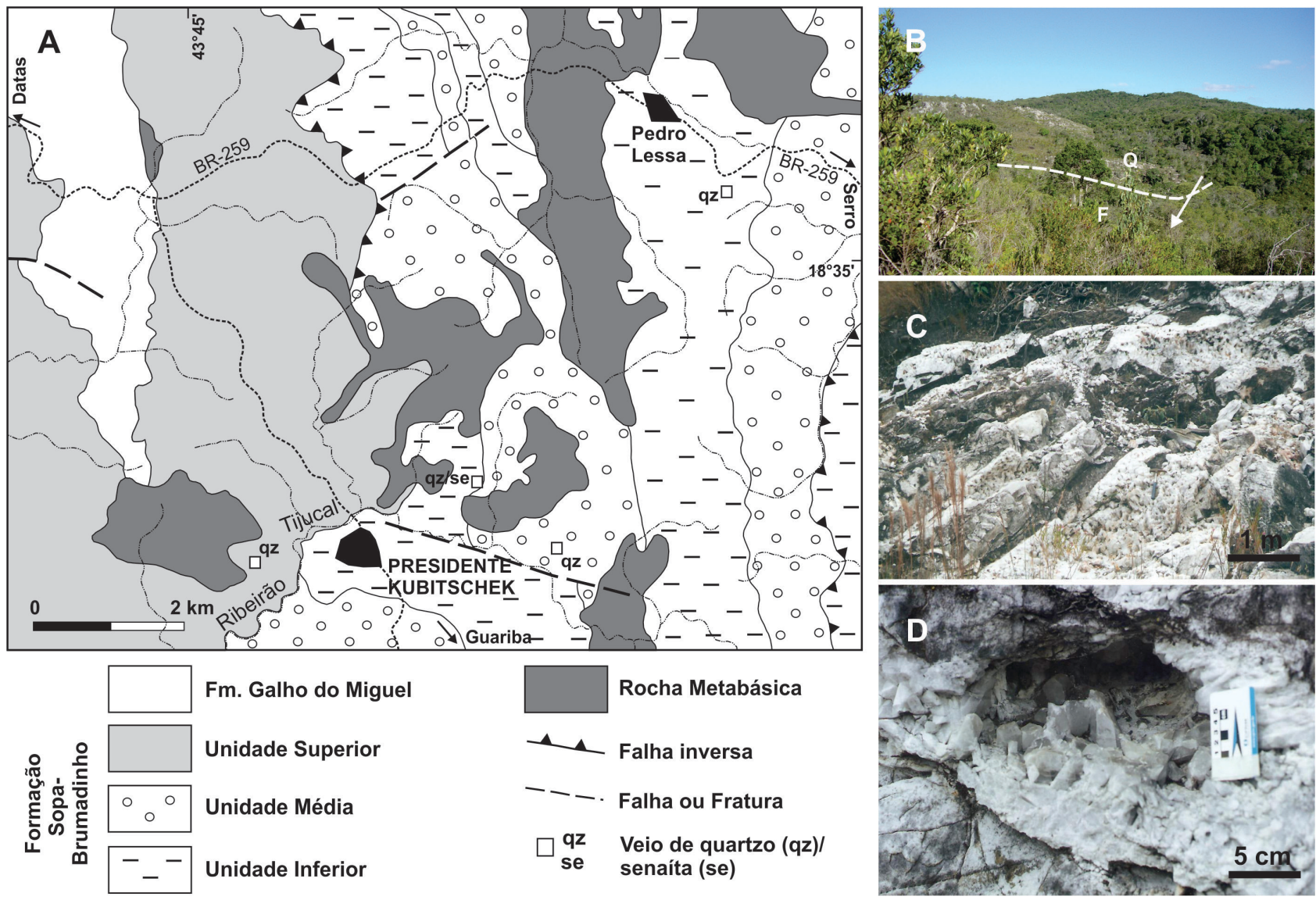

Figura 2. (A) Geologia da área do depósito de quartzo com senaíta a nordeste de Presidente Kubitschek, MG (parcial e modificada de Uhlein e Garcia, 1987); (B) Paisagem local destacando o contato entre filitos (F) e quartzitos (Q) da Formação Sopa-Brumadinho, com a seta indicando o local do depósito; (C) Enxame de veios de quartzo mineralizados; (D) Detalhe do veio principal, com "bucho" mostrando material melhor cristalizado. 
Chaves (1992), ao observar inicialmente o pico do estrôncio mais forte em relação ao do chumbo (Figura 3), indicando maior abundância do primeiro elemento, comprovada também através de análises semiquantitativas em MEV-EDS.

A geologia da área de ocorrência do mineral foi levantada por Chaves et al. (1988), onde se reconheceram quartzitos médios a grossos, pertencentes à Unidade Média da Formação Sopa-Brumadinho, empurrados sobre rochas metapelíticas da Unidade Superior desta formação (Figura 3A). Conforme Cassedanne (1986) e Cassedanne e Chaves (1990), ocorre na área um enxame de veios de quartzo associados, em que o maior destes está alojado numa zona de fraturamento de direção aproximada $\mathrm{N} 60^{\circ} \mathrm{W}$, possuindo entre 1,0 e $2,5 \mathrm{~m}$ de largura.

A porção oeste dessa zona de fraturamento (GPS-UTM 650715/7937740) foi lavrada manualmente por cerca de $50 \mathrm{~m}$ em escavações irregulares (Figura 3B), predominando quartzo leitoso e maciço; em sua parte central um corte $6 \mathrm{~m}$ de extensão por $3 \mathrm{~m}$ de profundidade mostrou um stockwork de veios irregulares com drusas de quartzo e quartzo "clorítico", explorados para coleção. Pequenas cavidades e fissuras dispersas, sem direção preferencial, estão preenchidas por cristais de quartzo de uma geração mais recente, na qual rutilo, anatásio e crichtonita são encontrados in situ.

Tais autores identificaram quatro hábitos principais nas amostras de crichtonita (Figura 3C, D): (a) romboedros pseudocúbicos com dimensões milimétricas; (b) cristais complexos, geminados, lembrando dolomita geminada da Suíça; (c) cristais tabulares com até $1 \mathrm{~cm}$, frequentemente cobertos por rutilo acicular; e (d) cristais "esponjosos", corroídos, que representam o tipo mais abundante.

Os primeiros dados químicos fornecidos por Cassedanne (1986), quando ainda se considerava tal material como "senaíta", limitaram-se a uma análise semiquantitativa por
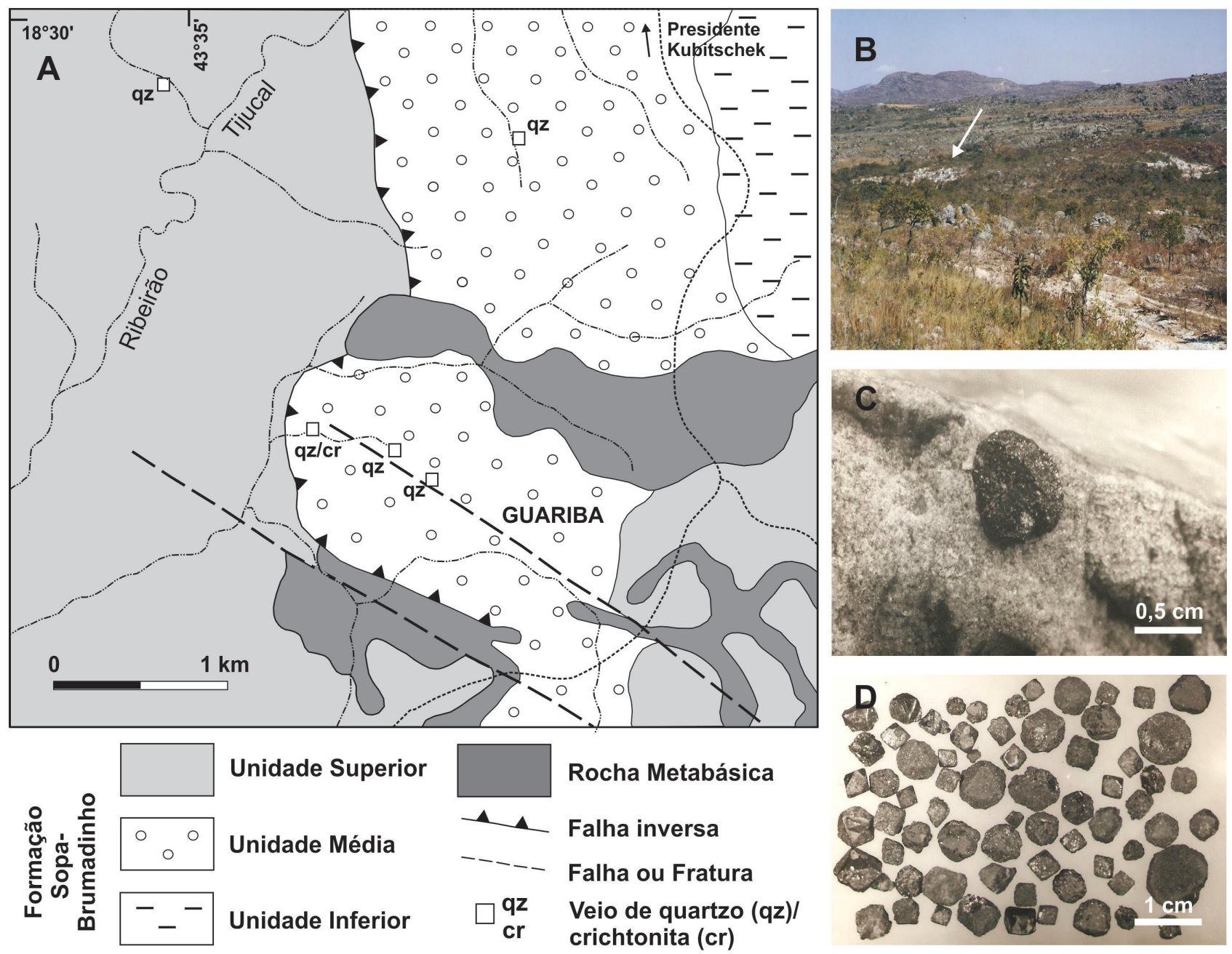

Figura 3. (A) Geologia da área do depósito de quartzo com crichtonita da Fazenda Guariba, a leste de Presidente Kubitschek (MG); (B) Vista geral da área, com a seta destacando o local do veio; (C) Detalhe de um cristal de crichtonita sobre quartzo leitoso; (D) Lote com grãos de crichtonita extraídos do veio, destacando cristais com as quatro formas preferenciais conforme o texto. 
fluorescência de raios $\mathrm{X}$, que mostrou teores de $\mathrm{Ti}, \mathrm{Fe}$ e Mn excedendo $10 \%$ (o que no caso do Mn está certamente incorreto); $\mathrm{Pb}, \mathrm{Sr}$ e Y entre 1 e 10\%; além de traços de La, $\mathrm{Cu}, \mathrm{Gd}, \mathrm{V}, \mathrm{Nd}$ e Th. As análises químicas com microssonda eletrônica e LA-ICP-MS que definiram o mineral como crichtonita (média de 20 amostras), realizadas por Foord et al. (1994), bem como novas análises, são apresentadas na Tabela 3, em que se observa notável concordância entre os dados. Com base nos dados ora obtidos, a fórmula empírica calculada do mineral é $\left(\mathrm{Sr}_{0,53} \mathrm{~Pb}_{0,38}\right)_{\Sigma 0,91}\left(\mathrm{Mn}_{0,48} \mathrm{Y}_{0,33} \mathrm{Sc}_{0,10) \Sigma 0,91}\right.$ $\left(\mathrm{Fe}^{2+}{ }_{1,90} \mathrm{Zn}_{0,10}\right)_{\sum 2,00}\left(\mathrm{Ti}_{13,58} \mathrm{Fe}^{3+}{ }_{4,42}\right)_{\sum 18,00} \mathrm{Fe}^{2+}{ }_{0,28} \mathrm{O}_{38}$.

Logo após a publicação desses trabalhos pertinentes, a zona rica em veios com crichtonita foi inteiramente detonada por garimpeiros e comerciantes de minerais de coleção da região de Governador Valadares (MG).

\section{Almeidaíta $\left[\mathrm{PbZn} \mathrm{n}_{2}(\mathrm{Mn}, \mathrm{Y})\left(\mathrm{Ti}, \mathrm{Fe}^{3+}\right)_{18} \mathrm{O}_{37}(\mathrm{OH}, \mathrm{O})\right]$ ou $\left[\mathrm{Pb}(\mathrm{Mn}, \mathrm{Y}) \mathrm{Zn}_{2}\left(\mathrm{Ti}, \mathrm{Fe}^{3+}\right)_{18} \mathrm{O}_{36}(\mathrm{O}, \mathrm{OH})_{2}\right]$}

A redefinição da senaíta de Presidente Kubitschek como crichtonita e a suspeição sobre a senaíta rica em Zn de Datas (conforme Foord et al., 1984) levaram os autores a realizarem novas análises geoquímicas com microssonda eletrônica sobre outras amostras de aspecto semelhante da região serrana do Espinhaço. Tais análises, conforme anteriormente visto, conduziram à descoberta do primeiro depósito de senaíta in situ, mas outras amostras coletadas na Bahia, quando analisadas, revelaram-se ainda mais surpreendentes. As composições químicas fornecidas são devidas a MINDAT (2016) e a Menezes Filho et al. (2015), respectivamente.

Nas proximidades de Novo Horizonte, borda oeste da Chapada Diamantina, são explorados desde a década de 1990 veios de quartzo ricos em drusas ou monocristais de quartzo com inclusões de rutilo e/ou hematita especular, cuja excepcionalidade tornou tal região uma referência mundial para amostras destes tipos (Menezes Filho e Chaves, 2007 - Figura 4A). Associados aos mesmos veios aparecem ainda monazita-(Ce), monazita-(La), bastnaesita-(La), rabdofânio-(La) e xenotímio-(Y) (todos comprovados através de análises com microssonda eletrônica), além de um mineral cinzento, de brilho submetálico, até então tratado genericamente como "hematita" pelos garimpeiros e comerciantes locais.

Estudos detalhados sobre esse material entre 2012-2013 (ano da aprovação do mineral pela IMA - International Mineralogical Association) conduziram à recente descoberta da almeidaíta, um dos mais novos minerais do grupo da crichtonita, por um grupo de pesquisadores nacionais e estrangeiros (Menezes Filho et al., 2015), no qual o primeiro autor do presente trabalho faz parte. É o $61^{\circ}$ mineral brasileiro, e seu nome foi dado em homenagem ao ilustre geólogo e professor Fernando Flávio Marques de Almeida (1916-2013), que chegou a conhecer tal descoberta e ser presenteado com uma amostra do mineral pelos autores (Figura 4B, C). A estrutura cristalina do mineral foi melhor definida em Rastsvetaeva et al. (2014).

Na região de Novo Horizonte afloram rochas metavulcânicas ácidas associadas à Formação Novo Horizonte, do Grupo Rio dos Remédios (Guimarães et al., 2005). O mineral foi originalmente descoberto em 2011, num rejeito de exploração abandonado de quartzo rutilado no Garimpo da Mula (Figura 5). Em novo trabalho de campo realizado em 2013, foram "desentupidas" as antigas escavações, encontrando-se também o mineral in situ, que ocorre disseminado no metariolito alterado, junto a um veio de quartzo métrico, de onde se supõe terem emanado as soluções hidrotermais mineralizantes (Chaves et al., 2016).

A almeidaíta se apresenta como cristais isolados, pretos submetálicos, de formas placoides e medindo até $30 \mathrm{~mm}$ x $30 \mathrm{~mm}$ x $6 \mathrm{~mm}$. Tal achado pode ser considerado admirável para um novo mineral, pelo porte excepcional dos cristais então encontrados (Figura 4B, C). Novos dados sobre a composição química deste mineral, caracterizado pela presença de ítrio no sítio "A", são apresentados na Tabela 4, observando-se notável concordância com os resultados obtidos por Menezes Filho et al. (2015). Com esses dados, a fórmula empírica calculada do mineral é $\left(\mathrm{Pb}_{0,65} \mathrm{Sr}_{0,10}\right)_{\Sigma 0,75}$ $\left(\mathrm{Mn}_{0,54} \mathrm{Y}_{0,47}\right)_{\Sigma 1,01} \mathrm{Zn}_{1,42}\left(\mathrm{Ti}_{13,02} \mathrm{Fe}^{3+}{ }_{4,98}\right)_{\Sigma 18,00} \mathrm{Fe}^{3+}{ }_{0,71} \mathrm{O}_{38}$.

Tabela 3. Novas análises com microssonda eletrônica em cinco amostras de crichtonita de Presidente Kubitschek Fazenda Guariba (médias de 4-5 spots por grão).

\begin{tabular}{lccccccccccc}
\hline Amostra & $\mathbf{S r O}$ & $\mathbf{T i O}_{\mathbf{2}}$ & $\mathbf{F e O}\left(^{*}\right)$ & $\mathbf{M n O}$ & $\mathbf{Z n O}$ & $\mathbf{Y}_{\mathbf{2}} \mathbf{O}_{\mathbf{3}}$ & $\mathbf{P b O}$ & $\mathbf{T R}_{\mathbf{2}} \mathbf{O}_{3}$ & $\mathbf{B a O}$ & $\mathbf{S c}_{\mathbf{2}} \mathbf{O}_{3}$ & $\mathbf{T o t a l}$ \\
\hline G5-g1 & 2,95 & 59,95 & 26,57 & 1,58 & 0,44 & 2,33 & 4,67 & $\mathrm{Nd}$ & $\mathrm{nd}$ & 0,35 & 99,28 \\
G5-g2 & 3,26 & 59,65 & 27,00 & 1,71 & 0,54 & 2,56 & 4,54 & $\mathrm{Nd}$ & $\mathrm{nd}$ & 0,35 & 99,61 \\
G5-g3 & 3,18 & 60,49 & 26,52 & 2,22 & 0,55 & 1,51 & 4,69 & $\mathrm{Nd}$ & $\mathrm{nd}$ & 0,39 & 99,55 \\
G5-g4 & 3,21 & 60,95 & 26,03 & 1,74 & 0,40 & 2,25 & 4,67 & $\mathrm{Nd}$ & $\mathrm{nd}$ & 0,33 & 99,58 \\
G5-g5 & 2,89 & 61,32 & 26,05 & 2,19 & 0,43 & 1,63 & 4,65 & $\mathrm{Nd}$ & $\mathrm{nd}$ & 0,37 & 99,53 \\
Média & 3,10 & 60,47 & 26,43 & 1,89 & 0,47 & 2,06 & 4,64 & $\mathrm{Nd}$ & $\mathrm{nd}$ & 0,36 & 99,42 \\
CR-F (**) & 2,90 & 59,40 & 26,70 & 1,81 & 0,50 & 1,95 & 3,45 & 2,65 & 0,27 & 0,40 & 100,03 \\
\hline
\end{tabular}

$\left({ }^{\star}\right)$ todo ferro contido como Fe ${ }^{2+} ;\left(^{* \star}\right)$ média das análises originais de Foord et al. (1994) a partir de amostras fornecidas pelo primeiro autor deste trabalho; nd - não determinado. 

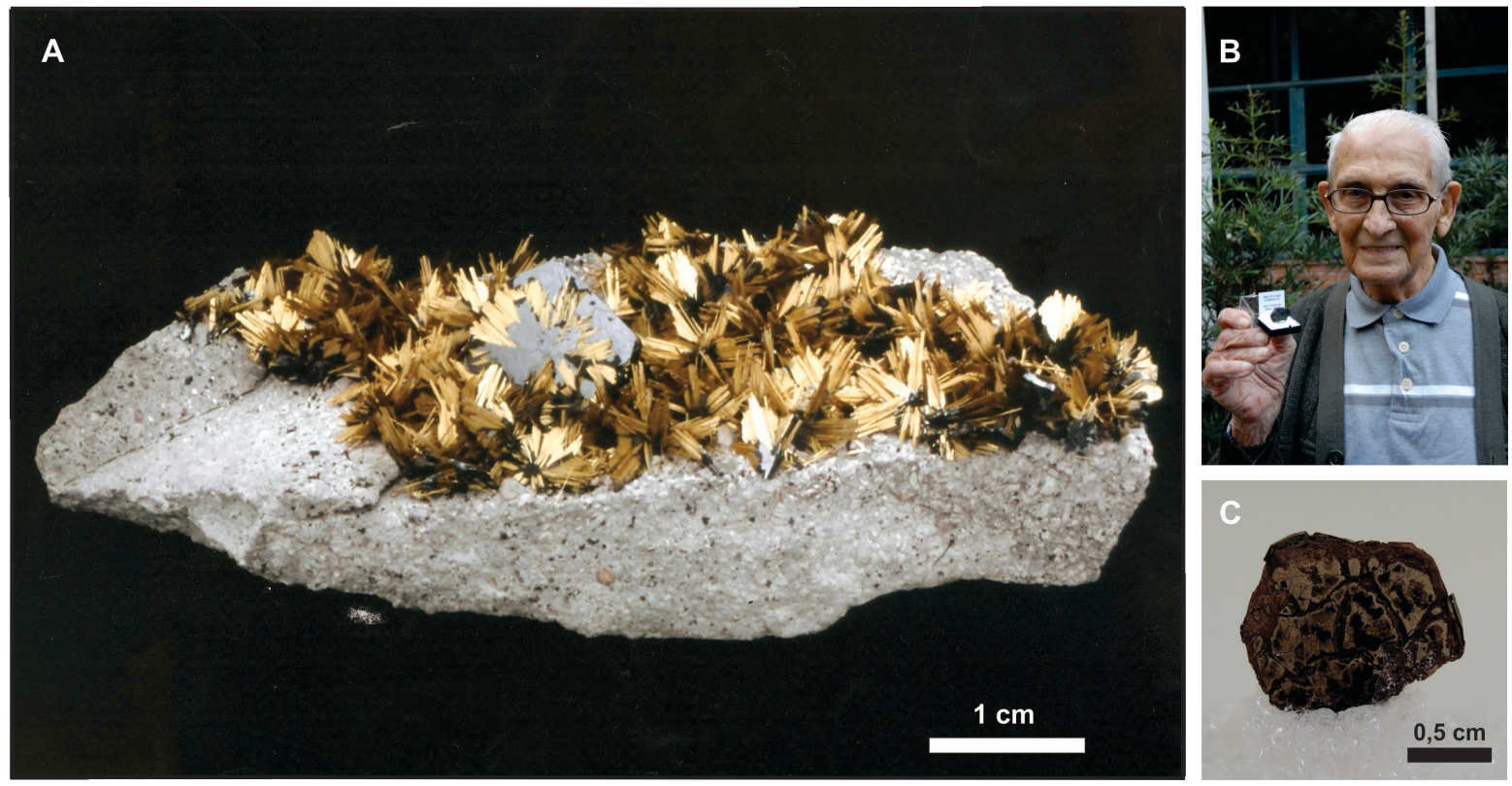

Figura 4. (A) Amostra excepcional de quartzo com hematita e rutilo sobre metariolito (Menezes Filho e Chaves, 2007); (B) Professor Fernando Flávio de Almeida ao ser presenteado com amostra de almeidaíta; (C) Detalhe de um cristal de almeidaíta da coleção do primeiro autor.

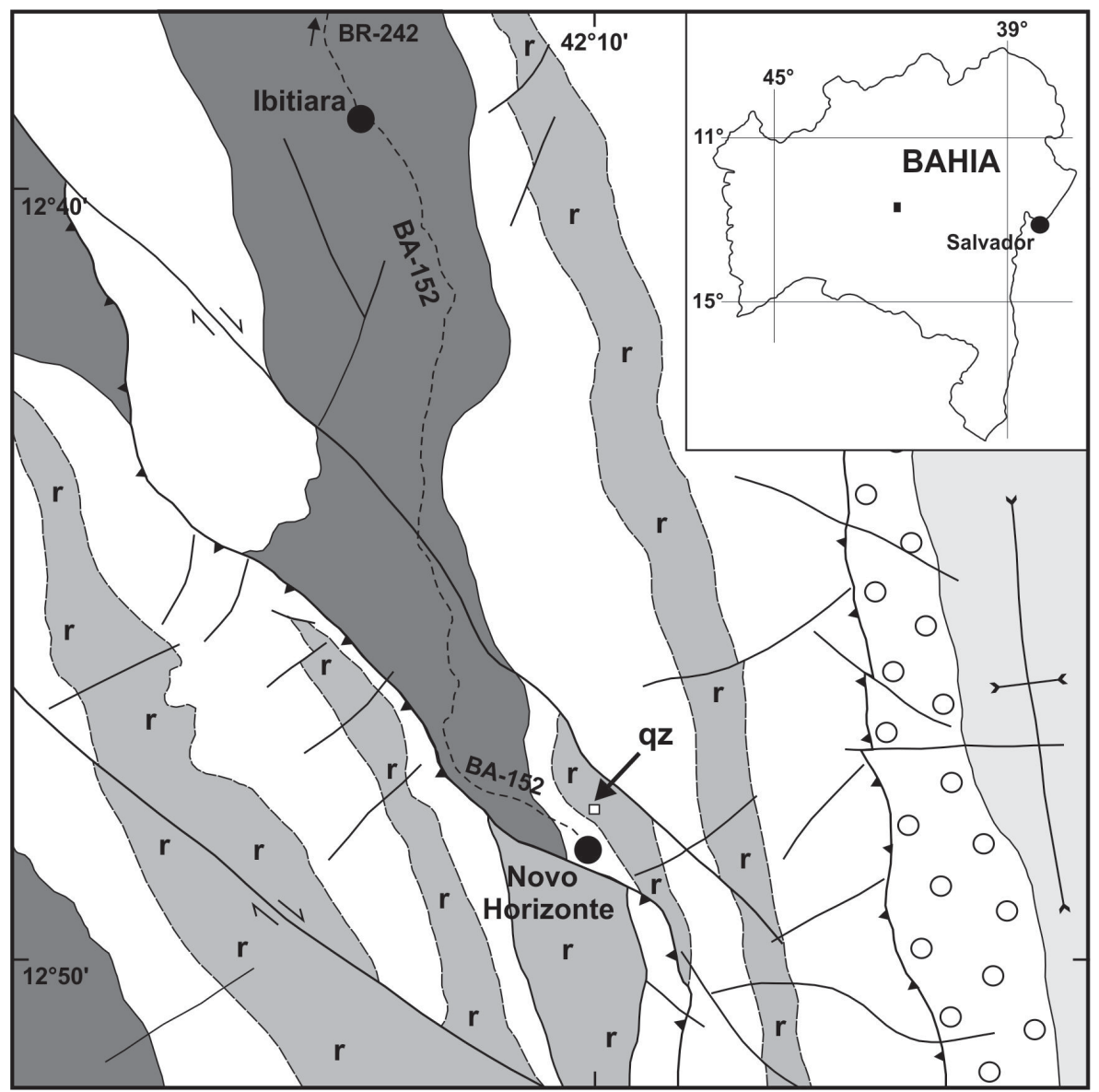

SUPERGRUPO ESPINHAÇO

GRUPO CHAPADA DIAMANTINA

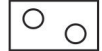

GRUPO

PARAGUAÇU

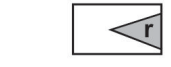

GRUPO RIO

DOS REMÉDIOS

( $r$-metariolito porfirítico)

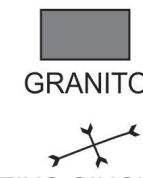

EIXO SINCLINAL

FALHA DE EMPURRÃO

FALHA TRANSPRESSIVA

FALHA OU FRATURA

$\square \mathrm{qz}$

VEIO DE QUARTZO

RUTILADO COM XENO-

TÍMIO-(Y) E ALMEIDAITA

ESTRÁDA

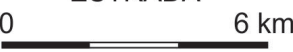

Figura 5. Geologia da região de Novo Horizonte (BA), com a seta destacando o local do Garimpo da Mula (qz), onde a almeidaíta foi descoberta. 
Tabela 4. Novas análises com microssonda eletrônica em três amostras de almeidaíta de Novo Horizonte (BA) - Garimpo da Mula (médias de 3-4 spots por grão).

\begin{tabular}{|c|c|c|c|c|c|c|c|c|c|c|c|}
\hline Amostra & Sro & $\mathrm{TiO}_{2}$ & $\mathrm{FeO}\left({ }^{*}\right)$ & $\mathrm{MnO}$ & Zno & $\mathrm{Y}_{2} \mathrm{O}_{3}$ & $\mathrm{PbO}$ & $\mathrm{CaO}$ & $\mathrm{TR}_{2} \mathrm{O}_{3}$ & $\mathrm{H}_{2} \mathrm{O}$ & Total \\
\hline AL-5-1 & 0,61 & 57,17 & 22,11 & 2,12 & 5,99 & 2,74 & 7,79 & $\mathrm{Nd}$ & nd & nd & 98,53 \\
\hline AL-5-2 & 0,64 & 56,02 & 22,82 & 2,04 & 6,86 & 3,09 & 8,01 & $\mathrm{Nd}$ & nd & nd & 99,48 \\
\hline AL-5-5 & 0,42 & 56,83 & 21,99 & 2,15 & 6,09 & 2,76 & 7,74 & $\mathrm{Nd}$ & nd & nd & 97,98 \\
\hline Média & 0,56 & 56,67 & 22,31 & 2,10 & 6,31 & 2,86 & 7,85 & $\mathrm{Nd}$ & nd & nd & 98,66 \\
\hline AL-M $\left({ }^{* *}\right)$ & 0,69 & 56,10 & 22,83 & 2,64 & 6,26 & 2,81 & 7,13 & 0,12 & 0,25 & 0,40 & 99,23 \\
\hline$A L-F\left({ }^{(* \star}\right)$ & 0,10 & 62,60 & 13,40 & 1,90 & 7,70 & 1,70 & 10,70 & $\mathrm{Nd}$ & 0,20 & nd & 98,30 \\
\hline
\end{tabular}

$\left(^{*}\right)$ todo ferro contido como Fe ${ }^{2+} ;\left(^{(\star}\right)$ análise original de Menezes Filho et al. (2015); $\left.{ }^{\star \star \star}\right)$ análise da erroneamente designada senaíta rica em Zn de Datas, conforme Foord et al. (1984); nd - não determinado.

Tabela 5. Análises com microssonda eletrônica de quatro spots sobre um mesmo grão de gramacciolíta-(Y) de Novo Horizonte (BA) - localidade desconhecida.

\begin{tabular}{lcccccccccc}
\hline \multicolumn{1}{c}{ Amostra } & $\mathbf{S r O}$ & $\mathbf{T i O}_{\mathbf{2}}$ & $\left.\mathbf{F e O} \mathbf{*}^{*}\right)$ & $\mathbf{M n O}$ & $\mathbf{Z n O}$ & $\mathbf{Y}_{\mathbf{2}} \mathbf{O}_{\mathbf{3}}$ & $\mathbf{P b O}$ & $\mathbf{U O 2}$ & $\mathbf{T r}_{\mathbf{2}} \mathbf{O}_{\mathbf{3}}$ & $\mathbf{T o t a l}$ \\
\hline GRA-29-1 & 1,33 & 56,78 & 23,47 & 2,52 & 2,78 & 3,49 & 8,48 & $\mathrm{nd}$ & $\mathrm{nd}$ & 98,85 \\
GRA-29-2 & 1,25 & 56,88 & 23,71 & 2,48 & 2,84 & 3,17 & 7,97 & nd & nd & 98,30 \\
GRA-29-3 & 1,27 & 57,70 & 23,20 & 2,49 & 2,80 & 3,44 & 8,84 & nd & nd & 99,74 \\
GRA-29-5 & 1,27 & 57,80 & 23,87 & 2,40 & 2,92 & 3,66 & 8,68 & nd & nd & 100,60 \\
Média & 1,28 & 57,29 & 23,56 & 2,47 & 2,84 & 3,44 & 8,49 & nd & nd & 99,37 \\
GRA-O (**) & 1,56 & 59,47 & 24,14 & 1,45 & 0,99 & 3,03 & 7,53 & 0,33 & 1,04 & 99,54 \\
\hline
\end{tabular}

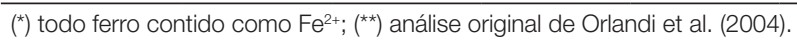

Em relação à senaíta rica em Zn de Foord et al. (1984), de fato também uma crichtonita, reconheceu-se os valores relativamente mais altos de $\mathrm{Ti}, \mathrm{Zn}$ e $\mathrm{Pb}$, e mais baixos de $\mathrm{Sr}$, Fe e Mn, indicando a provável influência de processos de isomorfismo.

\section{Gramacciolíta-(Y) $\left[(\mathrm{Pb}, \mathrm{Sr})(\mathrm{Y}, \mathrm{Mn}) \mathrm{Fe}^{3+}{ }_{2}\left(\mathrm{Ti}, \mathrm{Fe}^{3+}\right)_{18} \mathrm{O}_{38}\right]$}

Os importantes achados de senaíta primária e almeidaíta levaram também à checagem de toda a coleção de amostras de "hematita" procedentes de Novo Horizonte (BA) da coleção particular de Menezes Filho. A maioria das 15 amostras examinadas possuía crescimento epitaxial de rutilo, exceto uma, que constituía um cristal perfeito, geminado, tabular, preto metálico, medindo $1,8 \mathrm{~cm}$, incrustado sobre um agregado de cristais de quartzo rutilado. Uma pequena porção desse cristal foi especialmente extraída para análises, mostrando picos de EDS dos mesmos elementos principais da almeidaíta (óxido de $\mathrm{Pb}-\mathrm{Ti}-\mathrm{Fe}-\mathrm{Y}-\mathrm{Mn}$ ).

Entretanto, a análise quantitativa por microssonda eletrônica (WDS) feita em 2012 identificou um teor de ítrio maior que o de zinco (Tabela 5). Posteriormente, análise de difratometria de raios $\mathrm{X}$ em monocristal comprovou que se tratava da raríssima espécie gramaccioliíta-(Y) (Prof. N. Chukanov, 2013, em comunicação escrita aos autores); é a primeira descrição deste mineral no Brasil e o terceiro país onde ele é encontrado (além de Itália e Grécia - MINDAT, 2016). Quanto à composição química fornecida na descrição original do mineral na Itália (Orlandi et al., 2004), observam-se os valores mais expressivos de $\mathrm{Mn}$ e $\mathrm{Zn}$, e menores de $\mathrm{Sr}$ e Ti. Assim como no caso da almeidaíta, a presença mais significativa de $\mathrm{Zn}$ deve indicar a ocorrência de solução sólida. A fórmula empírica calculada é $\left(\mathrm{Pb}_{0,70} \mathrm{Sr}_{0,23}\right)_{\Sigma 0,93}$ $\left(\mathrm{Y}_{0,56} \mathrm{Mn}_{0,44}\right)_{\Sigma 1,00}\left(\mathrm{Fe}^{2+}{ }_{1,21} \mathrm{Zn}_{0,64}\right)_{\Sigma 1,85}\left(\mathrm{Ti}_{13,19} \mathrm{Fe}^{3+}{ }_{4,81}\right)_{\Sigma 18,00} \mathrm{Mn}_{0,21} \mathrm{O}_{38}$.

Infelizmente, não é ainda possível determinar a procedência exata de tal amostra, visto que ela foi adquirida na década de 1980. Novos estudos deverão ser conduzidos na região de Novo Horizonte a esse respeito.

\section{CONSIDERAÇÕES FINAIS}

Sem dúvida, ficou demonstrado que os veios de quartzo encontrados na Serra do Espinhaço são importantes fontes de espécies do grupo da crichtonita do "tipo hidrotermal" (Tabela 1), juntamente com outros minerais ricos em titânio. De tal modo, esses veios possuem forte potencial para a descrição de outros minerais do mesmo grupo, ou mesmo novas espécies ainda desconhecidas. As caracterizações químicas e detalhamentos geológicos integrados sobre os quatro minerais descritos - senaíta, crichtonita, almeidaíta e gramaccioliíta-(Y) - são importantes por constituírem suas primeiras identificações em nível nacional, enfatizando que a senaíta só havia sido identificada em depósitos aluvionares.

Interessante também observar e ressaltar um fator que inclusive pode conduzir a futuros estudos, relacionando-se à fonte de diversos elementos químicos "anômalos" na constituição dos litotipos básicos da Serra do Espinhaço quartzitos e filitos -, tais como $\mathrm{Sr}, \mathrm{Pb}, \mathrm{Zn}, \mathrm{Y}$ e Terras Raras. Na região da Chapada Diamantina (BA), rochas vulcânicas ácidas têm sido bem caracterizadas na porção basal do Supergrupo Espinhaço (Formação Novo Horizonte), as 
quais provavelmente constituíram a fonte de tais elementos. Na região do Espinhaço Meridional, no entanto, essas rochas são ainda mal conhecidas devido ao metamorfismo mais intenso, ou foram erodidas antes da deposição das unidades basais desse supergrupo.

Integrando-se os dados sobre os minerais do grupo da crichtonita da Serra do Espinhaço, algumas conclusões podem ser realçadas: (1) Senaíta: comprovou-se que ela é proveniente de veios de quartzo, e que a distribuição de tais depósitos é bastante extensa, já que o mineral é encontrado em aluviões distantes de Presidente Kubitschek, como em Datas, Serro e Extração (Hussak e Prior, 1898; Metelo, 1968; Cassedanne, 1986). (2) Crichtonita: possui área de ocorrência que parece ser mais restrita, uma vez que sua descrição ainda se limita ao depósito inicialmente reportado em Chaves (1992). (3) Almeidaíta: a descoberta recente e surpreendente deste mineral em Novo Horizonte (Menezes Filho et al., 2015), apresentando dimensões tão significativas, abre caminho para que novos minerais deste grupo possam ser identificados. Além disso, como a senaíta rica em Zn de Datas (Foord et al., 1984) é uma almeidaíta, essa localidade agora constitui a segunda ocorrência conhecida do mineral. (4) Gramacciolíta-(Y): a descrição de tal espécie comprova a presunção anteriormente relatada. Esse mineral foi achado na mesma região baiana (década de 1980), muito antes, portanto, de sua descrição oficial ocorrida na Itália (Orlandi et al., 2004).

Em suma, acredita-se que, com estudos mineralógicos mais detalhados sobre minerais de aspecto semelhante encontrados em veios de quartzo da Serra do Espinhaço, muitas e novas descobertas mineralógicas possam ainda ocorrer.

\section{REFERÊNCIAS}

Back, M. E., Mandarino, J. A. (2008). Fleischer's glossary of mineral species 2008. Tucson: Mineralogical Record Inc.

Cassedanne, J. P. (1986). Senaite from the Fazenda Guariba, Minas Gerais, Brazil. The Mineralogical Record, 17(3), 191-195.

Cassedanne, J. P., Chaves, M. L. S. C. (1990). Ocorrências de senaíta na região de Presidente Kubitschek, Minas Gerais. REM - Revista da Escola de Minas, 44, 42-46.

Chaves, M. L. S. C. (1992). Crichtonita: um mineral raro descrito pela primeira vez no Brasil. XXXVII Congresso Brasileiro de Geologia, v. 2, 1-2. São Paulo: SBG.

Chaves, M. L. S. C., Brandão, P. R. G., Buhn, B. (2010). Monazita em veios de quartzo da Serra do Espinhaço Meridional (MG): mineralogia, geocronologia e implicações geológicas. Revista Brasileira de Geociencias, 40(4), 506-515.

Chaves, M. L. S. C., Buhn, B., Dias, C. H., Menezes Filho, L. A. D. (2016). U-Pb ages in xenotime-(Y) from a quartz vein deposit with almeidaite, a new Brazilian mineral from the Espinhaço mountain range (Bahia State, Brazil). Journal of South American Earth Sciences, In press.

Chaves, M. L. S. C., Dussin, I. A., Rudnitzki, B. D. (1988). Geologia e paleoambientes de sedimentação do Supergrupo Espinhaço na região do Alto Rio Paraúna (MG). $X X X V$ Congresso Brasileiro de Geologia, v. 2, 617-632. Belém: SBG.

Chemale Júnior, F., Dussin, I. A., Alkmim, F. F., Martins, M. S., Queiroga, G., Armstrong, R., Santos, M. N. (2012). Unravelling a Proterozoic basin history through detrital zircon geochronology: the case of the Espinhaço Supergroup, Minas Gerais, Brazil. Gondwana Research, 22(1), 200-206.

Dussin, T. M. (1994). Associations plutono-volcaniques de l'Espinhaço Méridional: un exemple d'évolution de la croûte protérozoïque. Tese (Doutorado). Orléans: Université d'Orléans.

Foord, E. E., Chaves, M. L. S. C., Lichte, F. E. (1994). Plumboan crichtonite from the Fazenda Guariba, Minas Gerais, Brazil. The Mineralogical Record, 25(2), 133-135.

Foord, E. E., Sharp, W. N., Adams, J. W. (1984). Zinc- and Y-group-bearing senaite from St Peters Dome, and new data on senaite from Dattas, Minas Gerais, Brazil. Mineralogical Magazine, 48(346), 97-106.

Grey, I. E., Lloyd, D. J., White Junior, J. S. (1976). The structure of crichtonite and its relationship to senaite. The American Mineralogist, 61(11-12), 1203-1212.

Guimarães, D. (1934). À margem de "Os Satéllites do Diamante", v. 2, 58 p. Belo Horizonte: Serviço Geológico do Estado de Minas Gerais. Monografia.

Guimarães, J. T., Martins, A. A. M., Andrade Filho, E. L., Loureiro, H. C., Arcanjo, J. B. A., Neves, J. P. N., Abram, M. B., Silva, M. G., Melo, R. C., Bento, R. V. (2005). Projeto Ibitiara-Rio de Contas: relatório final. Salvador: Convênio CBPM-SGB-CPRM.

Hussak, E., Prior, G. T. (1898). On senaite, a new mineral belonging to the ilmenite group, from Brazil. Journal of the Mineralogical Society, 12, 30-32.

Hussak, E. (1917). Os satéllites do diamante. Rio de Janeiro: Typografia do Jornal do Commercio.

Machado, N., Schrank, A., Abreu, F. R., Knauer, L. G., Almeida-Abreu, P. A. (1989). Resultados preliminares da geocronologia U-Pb na Serra do Espinhaço Meridional. $V$ Simpósio de Geologia de Minas Gerais, 171-174. Diamantina: SBG.

Menezes Filho, L. A. D., Chaves, M. L. S. C. (2007). Minerais de coleção: ciência, estética e mercado. Geonomos, 15(2), 65-73. 
Menezes Filho, L. A. D., Chukanov, N. V., Rastsvetaeva, R. K., Chukanov, S. M., Pekov, I. V., Chaves, M. L. S. C., Scholz, R., Atencio, D., Brandão, P. R. G., Romano, A. W., Oliveira, L. C. A., Ardisson, J. D., Krambrock, K., Moreira, R. L., Persiano, A. C., Richards, R. P. (2015). Almeidaite, $\mathrm{PbZn} 2(\mathrm{Mn}, \mathrm{Y})(\mathrm{Ti}, \mathrm{Fe} 3+)$ 18O37(OH,O), a new crichtonite-group mineral, from Novo Horizonte, Bahia, Brazil. Mineralogical Magazine, 79(2), 269-283.

Metelo, M. J. (1968). Contribuição ao estudo da senaíta. XXII Congresso Brasileiro de Geologia, 125-129. Belo Horizonte: SBG.

MINDAT. (2016). Acesso em 02 de maio de 2016, <http:// www.mindat.org $>$.

Orlandi, P., Pasero, M., Duchi, G., Olmi, F. (1997). Dessauite, $(\mathrm{Sr}, \mathrm{Pb})(\mathrm{Y}, \mathrm{U})(\mathrm{Ti}, \mathrm{Fe} 3+) 20$, a new mineral of the crichtonite group from Buca della Vena Mine, Tuscany, Italy. The American Mineralogist, 82(7-8), 807-811.
Orlandi, P., Pasero, M., Rotiroti, N., Olmi, F., Demartin, F., Moëlo, Y. (2004). Gramaccioliite-(Y), a new mineral of the crichtonite group from Stura Valley, Piedmont, Italy. European Journal of Mineralogy, 16(1), 171-175.

Rastsvetaeva, R. K., Chukanov, S. M., Chukanov, N. V., Menezes Filho, L. A. D. (2014). Crystal structure of almeidaite, a new mineral of the crichtonite group. Doklady Chemistry, 455(1-2), 53-57.

Uhlein, A., Garcia, A. J. V. (1987). Estratigrafia, sedimentologia e tectônica do Supergrupo Espinhaço na região de Presidente Kubitschek, MG. I Simpósio sobre Sistemas Deposicionais no Pré-Cambriano, 97-111. Ouro Preto: SBG.

Wülser, P. A., Brugger, J., Meisser, N. (2004). The crichtonite group minerals: a review of the classification. Bulletin Liaison SFMC, 16, 76-77. 